\title{
Modeling Glucose and Subcutaneous Insulin Dynamics in Critical Care
}

\author{
Ari Pritchard-Bell ${ }^{\mathrm{a}}$, Gilles Clermontt,b,c , Timothy D. Knab ${ }^{\mathrm{a}}$, John Maalouf ${ }^{\mathrm{a}}$, \\ Michael Vilkhovoy ${ }^{\mathrm{d}}$, Robert S. Parker ${ }^{\mathrm{a}, \mathrm{b}, \mathrm{c}, *}$ \\ ${ }^{a}$ Department of Chemical and Petroleum Engineering, Swanson School of Engineering, \\ University of Pittsburgh, Pittsburgh, PA 15261 \\ ${ }^{b}$ Department of Critical Care Medicine, University of Pittsburgh, Pittsburgh, PA 15261 \\ ${ }^{c}$ McGowan Institute for Regenerative Medicine, University of Pittsburgh, Pittsburgh, PA \\ 15219 \\ ${ }^{d}$ School of Chemical and Biomolecular Engineering, Cornell University, Ithaca, NY 14853
}

\begin{abstract}
Model-based decision support could be used to tailor insulin treatment to patients suffering from stress hyperglycemia, while avoiding hypoglycemia. This work combines a previously published glucose and insulin model with a subcutaneous insulin delivery model, herein simplified using Markov Chain Monte Carlo optimization and Kullback-Liebler distance, to capture fast-acting and regular insulin using two shared and one type-specific fitted parameter. Glucose data from a critical care population $(\mathrm{N}=48)$ receiving subcutaneous insulin are fit to within finger stick glucose measurement error of $5 \%$ using a regularized, time-varying parameter. The resulting virtual patient cohort provides a basis on which automated insulin delivery systems can be tested.
\end{abstract}

Keywords: Biomedical Systems, Insulin Sensitivity, Critical Care, Mathematical Modeling, Parameter Estimation, Virtual Patient

\section{Introduction}

Stress hyperglycemia occurs frequently in critical care patients and many of the harmful repercussions may be mitigated by maintaining glucose within a

\footnotetext{
* Corresponding author

Email address: rparker@pitt.edu (Robert S. Parker)
}

Preprint submitted to Control Engineering Practice

July 1, 2016

C) 2016. This manuscript version is made available under the Elsevier user license http://www.elsevier.com/open-access/userlicense/1.0/ 
glucose target zone (GTZ) using insulin and glucose administration. Since the landmark publication of the first Leuven study in 2001 [1], showing a decrease in mortality from $8 \%$ to $4.6 \%$ in over 1,500 patients where a GTZ was maintained, there have been many attempts to create improved paradigms for treatment of stress hyperglycemia [2, 3]. Despite the literature supporting a GTZ [4], several follow-up studies to the 2001 Leuven study showed limited benefit: morbidity but not mortality reduction in the case of the Leuven follow-up study in 2006 [5], or no change in outcome whatsoever as seen in both Glucontrol 6] and CREATE-ECLA [7]. The waning potential for controlling stress hyperglycemia was exacerbated in 2009 when a multicenter prospective study (NICE-SUGAR [8] ) of over 6,000 patients showed an increase in mortality in the group receiving intensive insulin treatment. Retrospective analysis of the NICE-SUGAR study [9] indicates that improved outcomes from glycemic control are overwhelmed by the increased risk of hypoglycemia and the accompanying increase in mortality associated with hypoglycemia [10, 11, when aggressive glucose control is employed. Inconsistencies in glycemic control protocols [12, as well as variation in GTZ outcomes for different ICU subpopulations [13, 14, 15], has contributed to the mixed success of GTZ and subsequent disagreement regarding treatment protocols using insulin in the ICU [16, 17].

A more accurate, personalized treatment that is tailored to an individual may significantly improve patient outcome. The most promising method to achieve better control using a personalized strategy is through the use of a model-based decision support system (DSS), wherein a mathematical patient model is coupled with a controller and user interface that provides for closed-loop control under the supervision of a clinician. Such systems have been extensively investigated for use in managing Type 1 diabetes [18, 19, 20, 21]. A critical aspect of a DSS is accurate understanding and modeling of the various underlying mechanisms leading to stress hyperglycemia, as increased biological resolution and more accurate modeling is a critical component of control 22. Creating a decision support system with semi-automated control architecture allows for GTZ consistency across many different ICUs, thereby reducing variability in treat- 
ment implementation. Decision support systems have shown promising results in the critical care population [23, 24, however, much of the error and subsequent failure of control comes from the failure to resolve inter- and intra-patient variations in glucose dynamics following insulin administration. A predictive model of glucose dynamics following insulin delivery with a small number of fitted parameters provides a parameter-centric way to quantify patient variability.

Stress hyperglycemia and its accompanying deleterious effects are primarily treated via insulin infusion. Subcutaneously injected insulin is a less invasive form of delivery used preferentially when patients are deemed stable enough to transition from intravenous administration in the ICU. Therefore, a controlrelevant, population-based mathematical model that describes the patient-scale dynamics of subcutaneously administered insulin for multiple insulin types is the focus of this work. While several mathematical models have been proposed to describe subcutaneous insulin delivery [25, 26, 27], the present focus is a low-order (state and parameter dimension) model that was readily tailored to individual patients by changing a small number of practically-identifiable model parameters based on readily available clinical data. Previously published models of subcutaneous insulin [25, 26, 27] also tend to use different mathematical structures for each type of infused insulin (e.g., rapid-acting, regular, lente, etc.). The present work included regular and rapid-acting insulin types, and focused on constructing a single-structure model with parameters specific to insulin-type.

To build a low-order, practically identifiable subcutaneous insulin model, a previously reduced model [28], originally selected from a literature review of subcutaneous insulin models [26], was further analyzed using published human data [29, 30, 31, 32]. The model was fit using a Markov Chain Monte Carlo (MCMC) parameter search to provide posterior distributions of the model parameters. Finally, the reduced model of subcutaneous insulin delivery was validated with patient data from an intensive care clinical database to construct a virtual patient cohort for in silico analysis and potential use in control system design. 


\section{Methods}

\subsection{Insulin Absorption Model and Reduction}

In our earlier work [28, a subcutaneous insulin absorption model from literature [26] was modified and reduced while fitting previously published data of regular [29, 30] and fast-acting [31, 32] insulin. Similar to the original review [26, Akaikes information criterion [33, 34] (AIC) was used to compare and select the model variation that best balances fitting error against over-parameterization. The original model, shown in Figure [1A was modified to replace the complementary fractions $p$ and $1-p$, with a kinetically driven compartment $\left(Q_{s c}(t)\right)$ and corresponding rate coefficients $k 1$ and $k 2$, respectively, governing the rate of material transfer into the two possible insulin transport channels to form the "Extended" model, as shown in Figure 13. This structure was subsequently truncated by removing the second, single compartment (lower) as a result of the observation that, in the case of fitting each insulin type, the two compartment partition (top) is more utilized (total fraction of insulin mass going through this channel is $99 \%$ for fast-acting insulin and $79 \%$ for regular insulin). The so-called Reduced model, is shown in Figure $1 \mathrm{C}$, where only one channel of insulin transport remains. Figure 1D shows the final modifications made to the model in this work.

Separate parameters were fit for both fast-acting and regular insulin. Model selection was performed using AIC to establish the model having the best tradeoff between complexity (as measured by the number of free parameters) and quality of fit (a weighted sum-of-squared error between model predictions and data). Table 1 shows the comparison between the three models investigated. The original model from [26] and the reduced model had the same number of fitted parameters, however, the structure of the reduced model, specifically the parameter $k_{a 3}$, resulted in a lower AIC for both regular and fast-acting insulin, as shown in Table 1. The reduced model with low AIC scores for both regular and fast-acting insulin was determined in 28 as a superior model to describe subcutaneous insulin dynamics for multiple insulin types. 
Original

A)

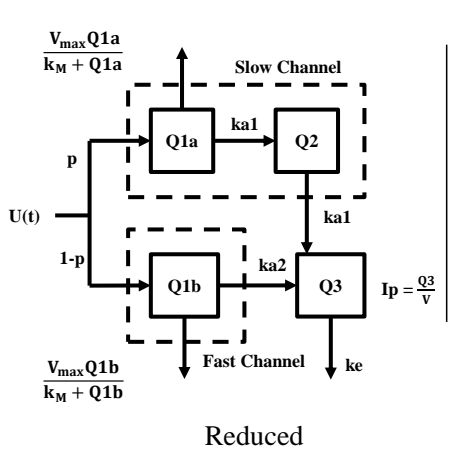

C)

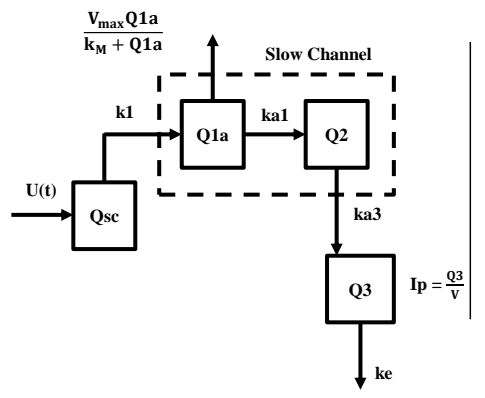

Extended

B)

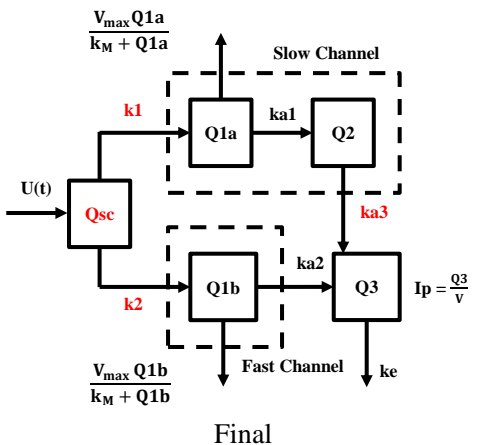

D)

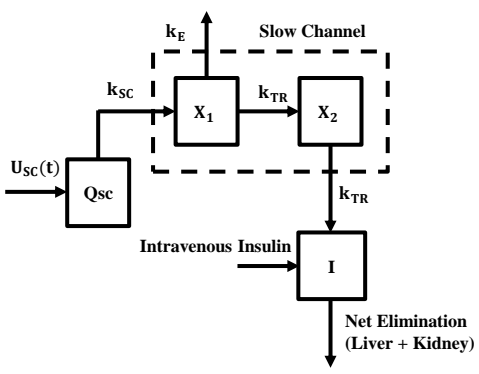

Figure 1: Reduction of the originally published model by Wilinska et al. [26] as described in [28] with the addition of panel D) showing the final model developed herein.

\subsection{Composite Model}

A previously published, clinically validated model of glucose and insulin dynamics [35] was combined with a version of the previously developed [28] reduced subcutaneous insulin model, which is herein further simplified to possess fewer fitted parameters (Figure 1D). The resulting composite model describes the dynamics of glucose and insulin, including exogenously administered glucose, insulin, and subcutaneous insulin. The mathematical description of the 
Table 1: Number of parameters, sum of squared error (SSE), and AIC scores for the three models fit with separate parameter values for both fast-acting and regular insulin.

\begin{tabular}{cccccc}
\multirow{2}{*}{ Model } & Parameters & \multicolumn{2}{c}{ SSE } & \multicolumn{2}{c}{ AIC } \\
& & Regular & Fast & Regular & Fast \\
\hline Wilinska et al. & 7 & 368.2 & 336.2 & 101.7 & 104.7 \\
Extended & 9 & 351.4 & 158.7 & 106.9 & 79.5 \\
Reduced & 7 & 356.7 & 186.0 & 100.6 & 79.8
\end{tabular}

subcutaneous insulin model is as follows.

$$
\begin{aligned}
\frac{d Q_{S C}(t)}{d t} & =U_{S C}(t)-k_{S C} Q_{S C}(t) \\
\frac{d X_{1}(t)}{d t} & =k_{S C} Q_{S C}(t)-k_{E} X_{1}(t)-k_{T R} X_{1}(t) \\
\frac{d X_{2}(t)}{d t} & =k_{T R} X_{1}(t)-k_{T R} X_{2}(t)
\end{aligned}
$$

The model we will use in this manuscript feeds a circulating glucose and insulin model modified from [35]:

$$
\begin{aligned}
\frac{d[I](t)}{d t}= & -n_{K}[I](t)-\frac{n_{L}[I](t)}{1+\alpha_{I}[I](t)}-n_{I}([I](t)-[Q](t)) \\
& +\frac{U_{e x}(t)+k_{T R} X_{2}(t)}{V_{I}} \\
\frac{d[Q](t)}{d t}= & n_{I}([I](t)-[Q](t))-n_{C} \frac{[Q](t)}{1+\alpha_{G}[Q](t)} \\
\frac{d[G](t)}{d t}= & -p_{G}[G](t)-S_{I}(t)[G](t) \frac{[Q](t)}{1+\alpha_{G}[Q](t)}+\frac{P(t)+E G P_{b}-C N S}{V_{G}}
\end{aligned}
$$

Here, subcutaneous insulin input, $U_{S C}(t)$, enters the model through the $Q_{S C}(t)$ state. In the simplified model, insulin mass travels through the states $X_{1}(t)$ and $X_{2}(t)$ and appears in the plasma compartment $I(t)$ with rate $k_{T R} X_{2}(t)$. This was added to the exogenous (intravenous) insulin administration term from [35], as both serve to increase the amount of circulating insulin. Subcutaneous insulin is removed from the compartment $X_{1}(t)$ at a rate $k_{E} X_{1}(t)$. This is shown schematically in Figure 1D.

When the subcutaneous model is added to the insulin-glucose model, the 


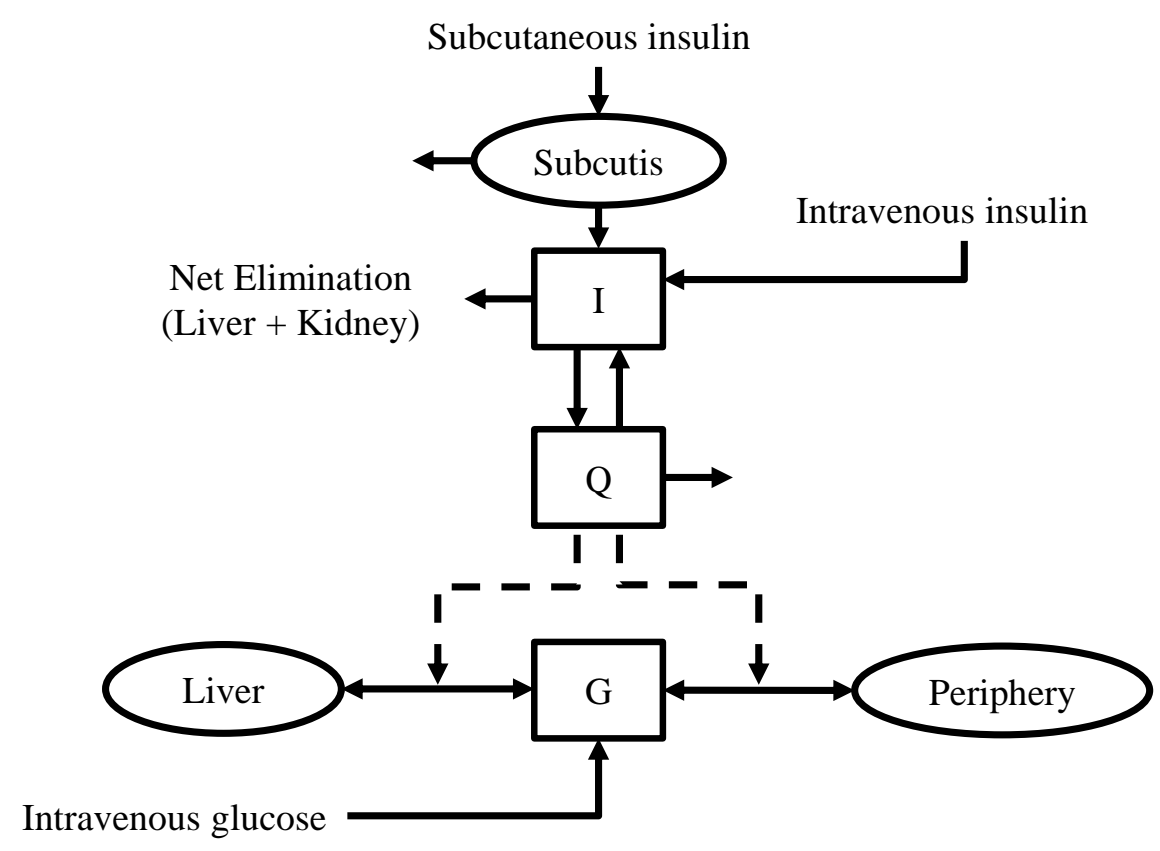

Figure 2: Composite model structure showing insulin and glucose plasma concentrations (squares), along with exogenous and endogenous sources of glucose and insulin.

state through which the two models are connected is the plasma insulin state, $I(t)$. In the previously described fitting and comparison of the isolated reduced subcutaneous model, the rate of insulin degradation is a first order linear degradation shown in Figure 1 C. However, when concatenating the two models, the insulin state has nonlinear elimination dynamics originating from the insulinglucose model, described in Equation (4). To reconcile this difference, the same insulin data [29, 30, 31, 32, are refit using the dynamic equations from Equation (4) to model insulin concentration.

\subsection{Parameter Estimation}

The parameter space was explored using an MCMC search with parallel tempering to provide posterior distributions for the subcutaneous insulin model 
parameters. The quantity $J$ is the energy function defined as:

$$
J(\boldsymbol{\theta})=\sum_{i=1}^{N}\left[y_{i}-y\left(t_{i}, \theta_{1} \ldots \theta_{M}\right)\right]^{2}
$$

Here, the variable $y_{i}$ is the measured data at time $t_{i}$. The model prediction is given by $y\left(t_{i}, \theta_{1}, \ldots, \theta_{M}\right)$, which depends on model parameters $\theta_{j}, j \in[1, M]$. $N$ is the number of data points, and $M$ is the total number of model parameters. Relative parameter steps were sampled from a Gaussian distribution and accepted according to the probability $\exp \left(\left(J_{\text {current }}-J_{\text {proposed }}\right) / \tau(k)\right)$, and parameter values swap with neighboring chains having temperatures, $\tau_{i}$ and $\tau_{j}$,

with probability $\exp \left(\left(J_{i}-J_{j}\right)\left(\frac{1}{\tau_{i}}-\frac{1}{\tau_{j}}\right)\right)$. The vector $\tau$ is the range of parallel chain temperatures $\tau(1) \ldots \tau(k)$. Equation (7) is the sum of squared error (SSE) between the data and model predictions, and this is proportional to the negative log-likelihood estimate of the model given the data.

\subsection{Data Workflow}

Patient data from the High-Density Intensive Care (HiDenIC) database at the University of Pittsburgh Medical Center (UPMC) were used to validate the composite model, which includes the subcutaneous module. A series of Python scripts were used to query the SQL database and construct tables of patient data matching the criteria needed for model validation. Data was extracted for 48 non-diabetic patients, with a length of stay in the ICU between 1 to 14 days, and between the years 2003 and 2009. Selection of the 48 patients was based on the existence of a window of approximately 72 hours during which the following three conditions were met: frequent (approximately 3-4 hour) finger stick glucose measurements [38, subcutaneous insulin administration, and no oral nutrition administration. These criteria allowed for the characterization of the delivered subcutaneous insulin effects on glucose levels in the absence of additional dynamic glucose processes such as gastric emptying or dynamics unique to diabetic patients. Intravenous glucose and insulin were also extracted over the interval of glucose measurements and modeled as direct inputs to Equations 6 and (4), respectively. 


\subsection{Virtual Patients}

For each extracted patient record, a metabolic profile was calculated in the form of a time-varying parameter, $S_{I}(t)$, the insulin sensitivity term from Equation (6), and a baseline constant $E G P_{b}$, similar to [36, 37]. The time-varying parameter $S_{I}(t)$ and constant $E G P_{b}$ were fit for each patient so that the combined effects of any exogenous inputs resulted in the model output matching the glucose values recorded in the data to within the error of the finger stick glucose measurement of $5 \%$ 38. While patients were selected based on available data, there may be some missing exogenous inputs in the data pulled from the clinical record. The resulting physiological effects of these missing data on blood glucose would result in additional fluctuation in the time-varying parameter $S_{I}(t)$. The parameter $S_{I}(t)$ is estimated every 5 minutes to approximate a continuous signal when compared to the hourly scale of glucose measurements in the database. Zero-order hold, per 5 minute changes in the metabolic profile, $S_{I}(t)$, were regularized by the parameter $\Gamma$, to smooth the $S_{I}(t)$ profile when fitting each 5 minute point, $t \in K$, parameter values of $S_{I}(t)$ to $N$ data points with $K \gg N$. The overall objective function for the optimization of each patient dataset is the minimization of $J$ from Equation (7) normalized by the number of glucose measurements and added to a regularization penalty on changes to $S_{I}(t)$, as follows:

$$
\left.\min _{S_{I}(t)} \frac{1}{N} J\left(S_{I}(t)\right)+\Gamma \sum_{i=1}^{N-1}\left(S_{I}\left(t_{i+1}\right)-S_{I}\left(t_{i}\right)\right)\right)^{2}
$$

Here, $\Gamma$ is the regularization parameter that penalizes the variance of the timevarying, patient-specific insulin sensitivity parameter $S_{I}(t)$. The virtual patient fitting procedure is summarized in Figure 3 . The regularization parameter $\Gamma$ was determined by fitting 48 patient profiles across a span of $\Gamma$ values and finding the value of $\Gamma$ that resulted in a mean absolute percent error (MAPE) per point of $5 \%$. This value was chosen to match the coefficient of variation precision threshold set for the blood glucose sensor [38]. The median $\Gamma$ value of $7.34 E 7$ was used to fit the virtual patients in generating the patient cohort. 


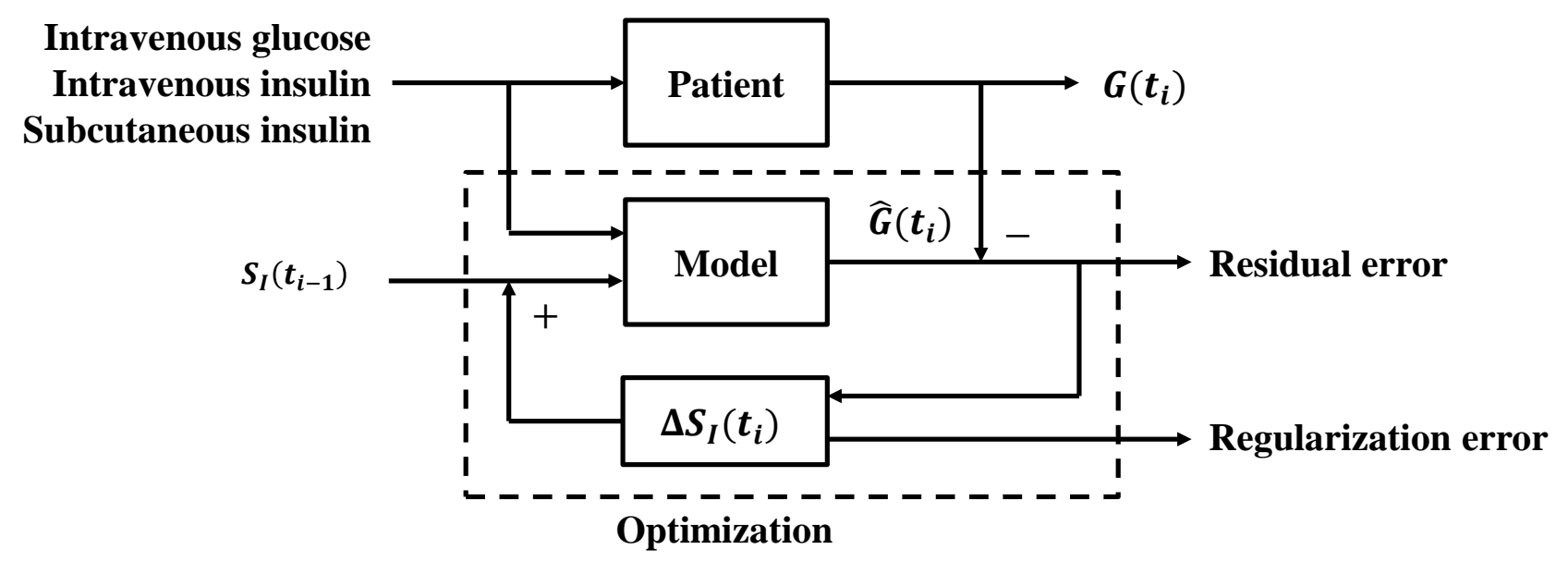

Figure 3: Block diagram for fitting metabolic profiles of patients by taking exogenous inputs and matching glucose output measurements through adjustment of insulin sensitivity, $S_{I}(t)$. Here, $G\left(t_{i}\right)$ is the glucose measurement from a patient at time $t_{i}$, which is fit by the model predicted glucose value $\hat{G}\left(t_{i}\right)$. The value $\Delta S_{I}\left(t_{i}\right)$ is the change in $S_{I}$ from $t_{i-1}$ to $t_{i}$.

Parameter estimation was performed using nonlinear least squares and solved using the interior point solver IPOPT [39] implemented in the Coopr/Pyomo package [40]. The model of ordinary differential equations was solved using orthogonal collocation on finite elements (5-minute duration for each element), with three Radau collocation points [41] per finite element.

\section{Results and Discussion}

\subsection{Parameter Space Reduction}

The number of parameters in the MCMC search space decreased in the simplified model case by replacing the original nonlinear degradation from state $X_{1}$ [26, 28, with a linear term, and fixing the parameters $k_{S C}$ and $k_{T R}$ to remain constant between insulin types. The resulting model is shown in Equation (1) to Equation (3), where only $k_{E}$ varies between insulin type. The parameter $k_{E}$ represents the effect of local degradation on subcutaneously delivered insulin before reaching the bloodstream. MCMC optimization was first run fitting three 
separate parameters $\left(k_{S C}, k_{T R}\right.$, and $\left.k_{E}\right)$ for each insulin type. The KullbackLeibler (KL) distances for each parameter were calculated between insulin types as in Equation (9), and are summarized in Table 2

$$
D(p \| q)=\sum_{x} p(x) \log _{2} \frac{p(x)}{q(x)}
$$

Here $D(p \| q)$ is the Kullback-Leibler distance between probability mass functions $p(x)$ and $q(x)$. The parameter $k_{E}$ was chosen to remain variable between insulin types due to having the largest KL distance between types. MCMC was run again to obtain the final parameter distributions. Model development and simplification was done using plasma insulin concentrations without information about the unobserved compartments present in the model. Therefore Equations (1) to (3) have no physiological meaning, and the model may not capture any additional insulin types without modifications. Additional data of various other insulin types are necessary to extend the model utility beyond regular and fast-acting insulin.

Table 2: Final Kullback-Leibler distances between parameter distributions fitting regular (R) and fast-acting (F) insulin.

\begin{tabular}{c|c|c|c} 
Distance & $k_{S C}$ & $k_{E}$ & $k_{T R}$ \\
\hline$D(R \| F)$ & $2.71 \mathrm{E}-1$ & $6.06 \mathrm{E}-1$ & $5.77 \mathrm{E}-1$ \\
$D(F \| R)$ & $2.87 \mathrm{E}-1$ & 1.74 & $8.51 \mathrm{E}-1$ \\
Total & $5.58 \mathrm{E}-1$ & 2.35 & 1.43
\end{tabular}

\subsection{Calibrating Model Parameters with MCMC Sampling}

The MCMC algorithm was used to compute the posterior parameter distributions after fitting the model (Equations (1) to (3p) to patient glucose data. The resulting subcutaneous insulin model is prepended to an existing model of glucose and insulin dynamics from the literature [35. The MCMC algorithm ran with good mixing for 1,000,000 sampled parameter steps. The initial 500,000 steps were truncated to account for burn-in and the final 500,000 steps were 
used to generate the parameter distributions shown in Figure 4. Human data

Posterior Parameter Distributions
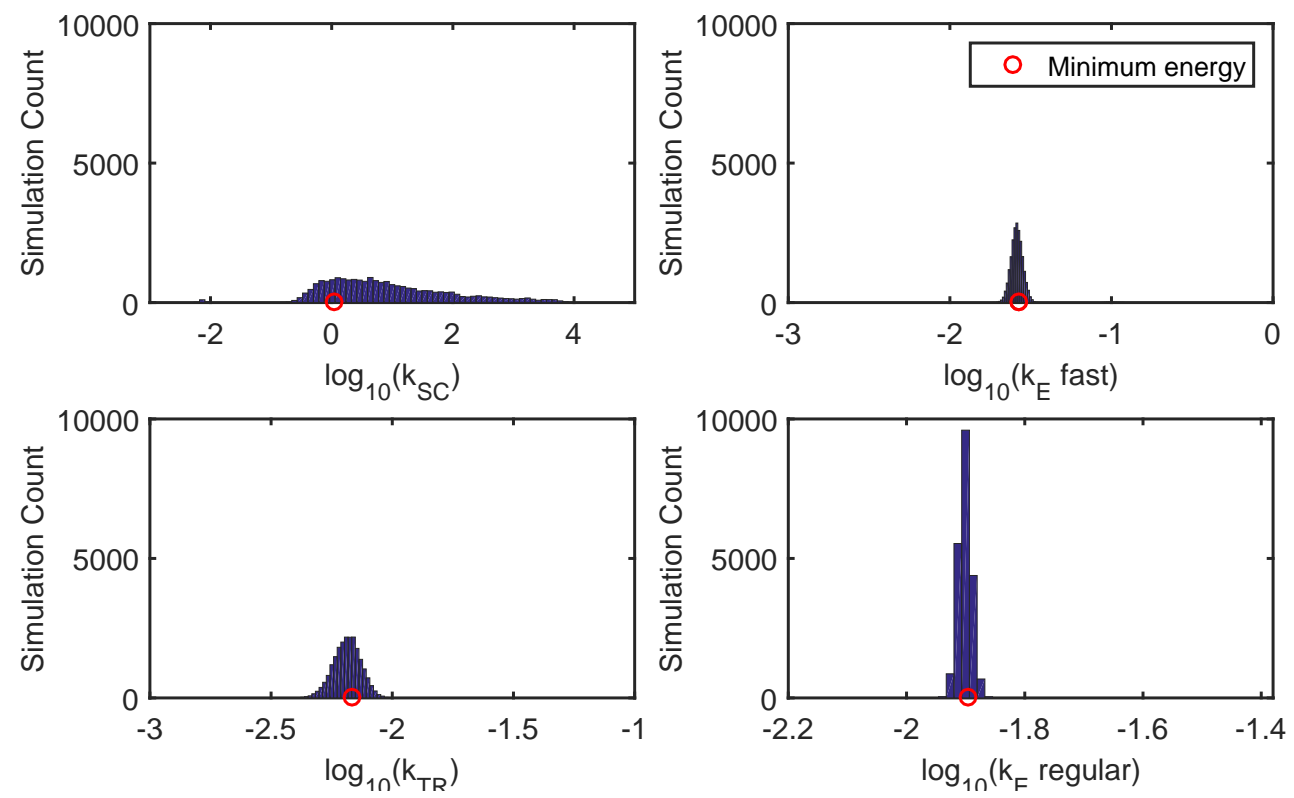

Figure 4: Subcutaneous insulin model parameter distributions for fast-acting and regular insulin found using MCMC optimization.

from [29, 30, 31, 32 were used to calibrate the model for fast-acting and regular insulin. To initialize Type 1 diabetic patients who had nonzero initial insulin concentrations from [31, 32, the model response to an insulin bolus of 5 units was simulated until the simulated insulin concentration (decreasing with time) matched that of the initial data point. The full state vector at this time point was used to initialize the model for parameter fitting purposes. This method attempts to capture the effect of insulin already on-board before the experiment was performed and data were collected. Failure to address the nonzero unobservable insulin states led to a pronounced drop in insulin immediately following the start of the simulation, before the simulated experimental subcutaneous bolus had reached the bloodstream. The resulting fits of the data using the minimum energy parameter sets found from MCMC are shown in Figures 5 and 6 . The 
final fitted model parameters are shown in Table 3 and the parameters used from [35] are shown in Table 4

Table 3: Final parameter values found using MCMC parameter optimization.

\begin{tabular}{cccc} 
Parameter & Units) & Regular & Fast \\
\hline$k_{E}$ & min $^{-1}$ & $2.68 \mathrm{E}-2$ & $6.82 \mathrm{E}-3$ \\
$k_{S C}$ & min $^{-1}$ & 1.08 & 1.08 \\
$k_{T R}$ & min $^{-1}$ & $1.27 \mathrm{E}-2$ & $1.27 \mathrm{E}-2$
\end{tabular}

Table 4: Parameters values from the original publication 35.

\begin{tabular}{ccc} 
Parameter & Units & Nominal [35] \\
\hline$a_{G}$ & $\frac{L}{m U}$ & $1.54 e-02$ \\
$n_{I}$ & $\mathrm{~min}^{-1}$ & $3.00 e-03$ \\
$n_{C}$ & $\mathrm{~min}^{-1}$ & $3.00 e-03$ \\
$n_{K}$ & $\mathrm{~min}^{-1}$ & $5.42 e-02$ \\
$n_{L}$ & $\mathrm{~min}^{-1}$ & $1.58 e-01$ \\
$a_{I}$ & $\frac{L}{m U}$ & $1.70 e-03$ \\
$V_{I}$ & $L$ & $3.15 e+00$ \\
$p_{G}$ & $m i n^{-1}$ & $6.00 e-03$ \\
$S I$ & $\frac{L}{m U \cdot m i n}$ & $3.00 e-04$ \\
$P$ & $\frac{m g}{m i n}$ & $0 e+00$ \\
$C N S$ & $\frac{m g}{m i n}$ & $5.40 e+01$ \\
$V_{G}$ & $d l$ & $1.33 e+02$
\end{tabular}

\subsection{Virtual Patient Metabolic Profiles}

The time-varying parameter $S_{I}(t)$ for each patient was regularized as in Equation (8), where the regularization parameter, $\Gamma$, was chosen as $7.3 E 7$ based on the distribution of $\Gamma$ values resulting in $5 \%$ average error per point. An example patient profile with modeled and measured glucose values, exogenous 
Fast-acting Insulin
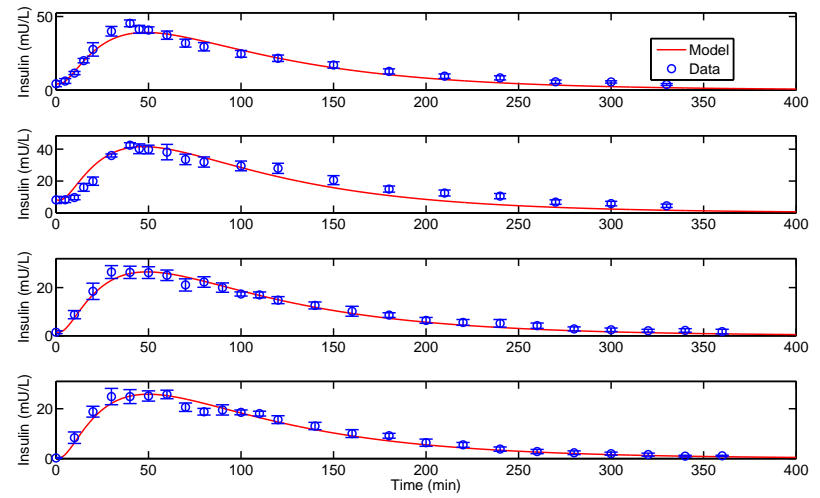

Figure 5: Plasma insulin model simulation (solid line) versus published data (circles, mean \pm standard error) for fast-acting subcutaneous insulin. Top panel: $10 \mathrm{U}$ insulin Lispro [31]. Second panel: $10 \mathrm{U}$ insulin Aspart 31]. Third panel: $7.1 \mathrm{U}$ insulin Lispro [32. Bottom panel: $7.1 \mathrm{U}$ insulin Aspart 32 . Note: y-axis changes between subfigures.

inputs, and $S_{I}(t)$ profile is shown in Figure 7. The results from fitting 48 patients from the HiDenIC database are summarized in Table 5. Overall, the time-varying parameter $S_{I}(t)$ and constant $E G P_{b}$ were able to capture the blood glucose profiles for individual patients to within an average absolute mean error $\left(\frac{1}{n} \sum_{i=1}^{n}\left[\frac{1}{m_{i}} \sum_{j=1}^{m}\left|\left[G_{i, j}\right]-\left[\hat{G}_{i, j}\right]\right|\right]\right)$ of $3.7 \mathrm{mg} / \mathrm{dL}$ per patient per data point to match that of the blood glucose sensor error [38, where $n$ is the total number of patients (48) and $m_{i}$ is the number of glucose measurements per patient in the data. Here $\left[\hat{G}_{i, j}\right]$ is the simulated glucose value, and the corresponding glucose measurement is $\left[G_{i, j}\right]$. Model-based analysis indicates that patients fall outside of the desired targeted blood glucose range of 80 to $130 \mathrm{mg} / \mathrm{dL}$ on average 12 $\%$ of the time.

\section{Summary}

Markov Chain Monte Carlo parameter optimization was used to consolidate from 4 parameters fit for each insulin type ( 8 total) to 3 parameters fit in total for the subcutaneous insulin infusion model. This parameter-efficient 
Regular Insulin
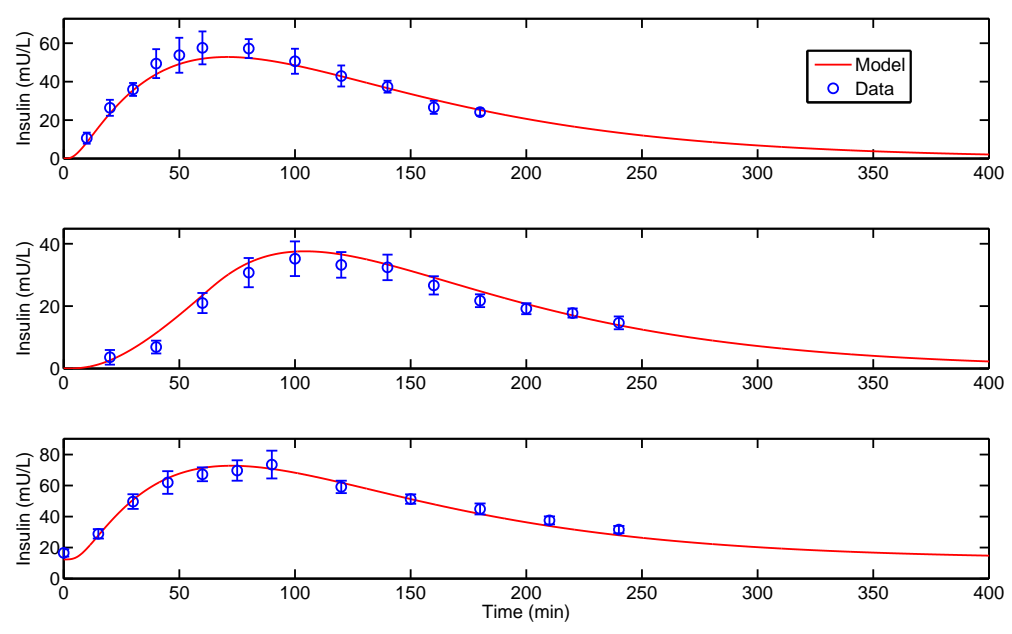

Figure 6: Plasma insulin model simulation (solid line) versus published data (circles, mean \pm standard error) for regular subcutaneous insulin. Top panel: 9 U bolus [30]. Middle panel: $6.8 \mathrm{U}$ continuous subcutaneous infusion over 60 minutes [30]. Bottom panel: $10 \mathrm{U}$ bolus 29]. Note: y-axis changes between subfigures. 
Patient 35
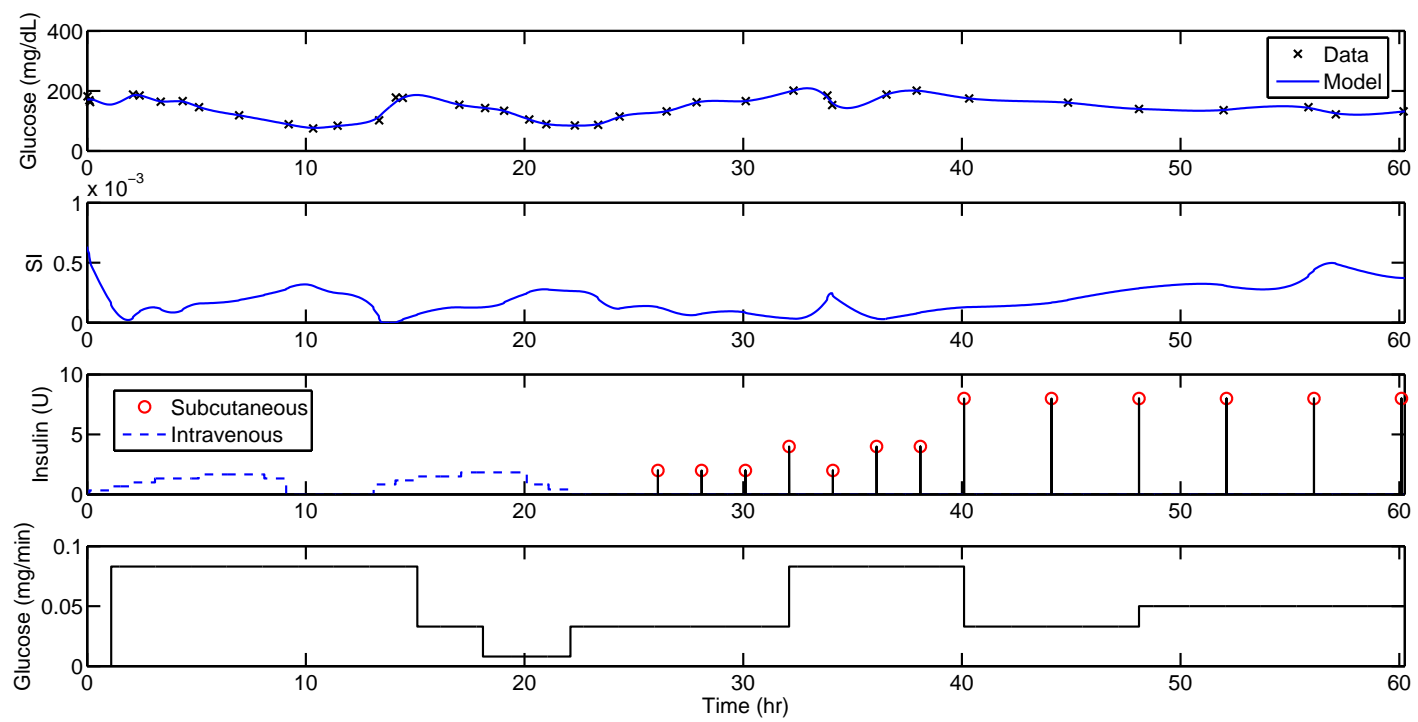

Figure 7: Representative patient fit by adjusting parameter $S_{I}(t)$. Top panel: Measured glucose values $(x)$ and model-predicted glucose (line). Second panel: Time-varying parameter $S_{I}(t)$. Third panel: Exogenous subcutaneous (black line, red circle) and intravenous (blue dashed line) insulin. Bottom panel: Exogenous intravenous glucose. 
Table 5: Results of fitting the $S_{I}(t)$ profile of 48 patients to match measured blood glucose. Note: $\left.{ }^{*}\right)$ the parameter $S_{I}(t)$ is lower bounded by zero when the recorded exogenous glucose input does not sufficiently account for the observed increase in blood glucose.

\begin{tabular}{lcccc} 
Quantity (units) & Mean & SD & Minimum & Maximum \\
\hline Mean absolute error $(\mathrm{mg} / \mathrm{dL})$ & 3.7 & 3.0 & 0.4 & 14.3 \\
Time in 80 to $130 \mathrm{mg} / \mathrm{dL}$ range $(\%)$ & 88.2 & 13.5 & 45 & 100 \\
$S_{I}(t)(\mathrm{L} / \mathrm{mU} / \mathrm{min})$ & $3.3 \mathrm{E}-4$ & $2.9 \mathrm{E}-4$ & $0.0^{*}$ & $2.3 \mathrm{E}-3$ \\
$E G P_{b}(\mathrm{mmol} / \mathrm{L} / \mathrm{min})$ & 1.7 & 0.65 & 1.0 & 4.9 \\
Subcutaneous insulin given $(\mathrm{U})$ & 67 & 44 & 20 & 214 \\
Patient data record length $(\mathrm{hr})$ & 66 & 7.9 & 37 & 72
\end{tabular}

structure fits $k_{S C}$ and $k_{T R}$ across insulin types while $k_{E}$ changes depending on the type of insulin administered. Virtual patients were constructed by fitting the composite model to intensive care unit data from patients who had received exogenous subcutaneous insulin in addition to intravenous glucose and insulin. One time-varying parameter, $S_{I}(t)$, and one constant parameter, $E G P_{b}$, were fit for each of 48 patients to form an individualized metabolic profile. Blood glucose measurements were captured using the recorded exogenous inputs as well as the fitted parameters, resulting in an average model error of $3.7 \mathrm{mg} / \mathrm{dL}$ per measurement per patient, to match blood glucose sensor error from [38, over an average time window of 66 hours. The composite model successfully simulates both regular and fast-acting subcutaneous insulin administration and captures blood glucose dynamics in a critical care population. One of the primary challenges in treating stress hyperglycemia in critical care is the intra- and inter-patient variations in glucose metabolism resulting from changes in insulin sensitivity. The fitted parameter profile, $S_{I}(t)$, calculated for each patient data set extracted from the critical care database forms a virtual patient cohort. Each unique, time-varying parameter profile can be used to simulate a realistic patient responding to exogenous inputs such as insulin and glucose administration in critical care. This forms a test platform for in silico controller design and 
optimization capable of utilizing subcutaneous insulin and expanding beyond strictly intravenous-based control.

\section{Acknowledgment}

Research reported in this publication was supported by the National Institute of Diabetes, Digestive and Kidney Disease (NIDDK) of the National Institutes of Health under award number R21-DK-92813. This work was supported partially by the Research Experiences for Undergraduates (REU) Program of the National Science Foundation under Award Number EEC-1156899.

\section{References}

[1] G. Van den Berghe, P. Wouters, F. Weekers, C. Verwaest, F. Bruyninckx, M. Schetz, D. Vlasselaers, P. Ferdinande, P. Lauwers, R. Bouillon, Intensive insulin therapy in critically ill patients, New England journal of medicine 345 (19) (2001) 1359-1367.

URL http://www.nejm.org/doi/full/10.1056/NEJMoa011300

[2] J. S. Krinsley, Effect of an intensive glucose management protocol on the mortality of critically ill adult patients, in: Mayo Clinic Proceedings, Vol. 79, Elsevier, 2004, pp. 992-1000.

URL http://www.sciencedirect.com/science/article/pii/ S002561961162572X

[3] J.-C. Preiser, P. Devos, G. Van den Berghe, Tight control of glycaemia in critically ill patients, Current Opinion in Clinical Nutrition \& Metabolic Care 5 (5) (2002) 533-537.

URL http://journals.lww.com/co-clinicalnutrition/Abstract/ 2002/09000/Tight_control_of_glycaemia_in_critically_ill.12.

aspx

[4] G. E. Umpierrez, S. D. Isaacs, N. Bazargan, X. You, L. M. Thaler, A. E. Kitabchi, Hyperglycemia: an independent marker of in-hospital mortality 
in patients with undiagnosed diabetes, The Journal of Clinical Endocrinology \& Metabolism 87 (3) (2002) 978-982.

URL http://press .endocrine.org/doi/abs/10.1210/jcem.87.3.8341

[5] G. Van den Berghe, A. Wilmer, G. Hermans, W. Meersseman, P. J. Wouters, I. Milants, E. Van Wijngaerden, H. Bobbaers, R. Bouillon, Intensive insulin therapy in the medical ICU, New England Journal of Medicine 354 (5) (2006) 449.

URL http://www . biomedcentral.com/content/pdf/cc5953.pdf

[6] J.-C. Preiser, P. Devos, S. Ruiz-Santana, C. Mélot, D. Annane, J. Groeneveld, G. Iapichino, X. Leverve, G. Nitenberg, P. Singer, J. Wernerman, M. Joannidis, A. Stecher, R. Chioléro, A prospective randomised multicentre controlled trial on tight glucose control by intensive insulin therapy in adult intensive care units: the glucontrol study, Intensive Care Medicine 35 (10) (2009) 1738-1748. doi:10.1007/s00134-009-1585-2.

URL http://link.springer . com/10.1007/s00134-009-1585-2

[7] S. R. Mehta, Effect of glucose-insulin-potassium infusion on mortality in patients with acute ST-segment elevation myocardial infarction: The CREATE-ECLA randomized controlled trial, JAMA 293 (4) (2005) 437. doi:10.1001/jama.293.4.437.

URL http://jama.jamanetwork.com/article.aspx?doi=10.1001/ jama.293.4.437

[8] S. Finfer, Intensive versus conventional glucose control in critically ill patients, New England Journal of Medicine 360 (13) (2009) 1283-1297. doi:10.1056/NEJMoa0810625

URL http://www .nejm.org/doi/abs/10.1056/NEJMoa0810625

[9] S. Finfer, Hypoglycemia and risk of death in critically ill patients, New England Journal of Medicine 367 (12) (2012) 1108-1118. doi:10.1056/ NEJMoa1204942,

URL http://www.nejm.org/doi/abs/10.1056/NEJMoa1204942 
[10] J. Hermanides, R. J. Bosman, T. M. Vriesendorp, R. Dotsch, F. R. Rosendaal, D. F. Zandstra, J. B. L. Hoekstra, J. H. DeVries, Hypoglycemia is associated with intensive care unit mortality*: Critical Care Medicine 38 (6) (2010) 1430-1434. doi:10.1097/CCM.0b013e3181de562c.

URL http://content.wkhealth.com/linkback/openurl?sid=WKPTLP: landingpage\&an $=00003246-201006000-00006$

[11] J. S. Krinsley, A. Grover, Severe hypoglycemia in critically ill patients: Risk factors and outcomes*:, Critical Care Medicine 35 (10) (2007) 2262-2267. doi:10.1097/01.CCM.0000282073.98414.4B

URL http://content.wkhealth.com/linkback/openurl?sid=WKPTLP: landingpage\&an $=00003246-200710000-00004$

[12] M. Wilson, J. Weinreb, G. W. S. Hoo, Intensive insulin therapy in critical care: A review of 12 protocols, Diabetes Care 30 (4) (2007) 1005-1011. doi:10.2337/dc06-1964.

URL http://care.diabetesjournals.org/cgi/doi/10.2337/ dc06-1964

[13] R. Tiruvoipati, B. Chiezey, D. Lewis, K. Ong, E. Villanueva, K. Haji, J. Botha, Stress hyperglycemia may not be harmful in critically ill patients with sepsis, Journal of Critical Care 27 (2) (2012) 153-158. doi:10.1016/j.jcrc.2011.06.011 URL http://linkinghub.elsevier.com/retrieve/pii/ S0883944111002504

[14] H. Hirasawa, Blood glucose control in patients with severe sepsis and septic shock, World Journal of Gastroenterology 15 (33) (2009) 4132. doi:10. $3748 /$ wjg. 15.4132

URL http://www.wjgnet.com/1007-9327/15/4132.asp

[15] B. W. Whitcomb, E. K. Pradhan, A. G. Pittas, M.-C. Roghmann, E. N. Perencevich, Impact of admission hyperglycemia on hospital mortality in various intensive care unit populations*:, Critical Care Medicine 33 (12) 
(2005) 2772-2777. doi:10.1097/01.CCM.0000189741.44071.25.

URL http://content.wkhealth.com/linkback/openurl?sid=WKPTLP:

landingpage\&an=00003246-200512000-00009

[16] P. E. Marik, J.-C. Preiser, Toward understanding tight glycemic control in the ICU: a systematic review and metaanalysis, CHEST Journal 137 (3) (2010) 544-551.

URL http://journal.publications.chestnet.org/article.aspx? volume $=137 \&$ issueno $=3 \&$ page $=544 \&$ etoc

[17] P. Parsons, P. Watkinson, Blood glucose control in critical care patients-a review of the literature, Nursing in critical care 12 (4) (2007) 202-210.

URL http://onlinelibrary.wiley.com/doi/10.1111/j.1478-5153. 2007.00225.x/full

[18] M. E. Wilinska, R. Hovorka, Simulation models for in-silico evaluation of closed-loop insulin delivery systems in type 1 diabetes, in: V. Marmarelis, G. Mitsis (Eds.), Data-driven Modeling for Diabetes, Lecture Notes in Bioengineering, Springer Berlin Heidelberg, 2014, pp. 131-149.

URL

http://link.springer.com/chapter/10.1007/

978-3-642-54464-4_6

[19] M. Breton, A. Farret, D. Bruttomesso, S. Anderson, L. Magni, S. Patek, C. D. Man, J. Place, S. Demartini, S. D. Favero, C. Toffanin, C. HughesKarvetski, E. Dassau, H. Zisser, F. J. Doyle, G. D. Nicolao, A. Avogaro, C. Cobelli, E. Renard, B. Kovatchev, o. b. o. T. I. A. P. i. S. Group, Fully integrated artificial pancreas in type 1 diabetes modular closed-loop glucose control maintains near normoglycemia, Diabetes 61 (9) (2012) 2230-2237. doi:10.2337/db11-1445.

URL http://diabetes . diabetes journals . org/content/61/9/2230

[20] S. J. Russell, F. H. El-Khatib, D. M. Nathan, K. L. Magyar, J. Jiang, E. R. Damiano, Blood glucose control in type 1 diabetes with a bihormonal bionic endocrine pancreas, Diabetes Care 35 (11) (2012) 2148-2155. doi: 
$10.2337 / \mathrm{dc} 12-0071$

URL http://care.diabetes journals .org/content/35/11/2148

[21] T. Battelino, J. Š. Omladič, M. Phillip, Closed loop insulin delivery in diabetes, Best Practice \& Research Clinical Endocrinology \& Metabolism doi:10.1016/j.beem.2015.03.001

URL http://www.sciencedirect.com/science/article/pii/ S1521690X15000196

[22] M. Morari, Robust process control, Prentice Hall, Englewood Cliffs, N.J, 1989.

[23] S. Eslami, A. Abu-Hanna, E. d. Jonge, N. F. d. Keizer, Tight glycemic control and computerized decision-support systems: a systematic review, Intensive Care Medicine 35 (9) (2009) 1505-1517. doi:10.1007/s00134-009-1542-0.

URL http://link.springer.com/article/10.1007/ s00134-009-1542-0

[24] L. Leelarathna, S. W. English, H. Thabit, K. Caldwell, J. M. Allen, K. Kumareswaran, M. E. Wilinska, M. Nodale, J. Mangat, M. L. Evans, others, Feasibility of fully automated closed-loop glucose control using continuous subcutaneous glucose measurements in critical illness: a randomized controlled trial, Crit Care 17 (2013) R159.

URL http://www.biomedcentral .com/content/pdf/cc12838.pdf

[25] G. Nucci, C. Cobelli, Models of subcutaneous insulin kinetics. a critical review, Computer Methods and Programs in Biomedicine 62 (3) (2000) 249-257. doi:10.1016/S0169-2607(00)00071-7.

URL http://www.sciencedirect.com/science/article/pii/ S0169260700000717

[26] M. Wilinska, L. Chassin, H. Schaller, L. Schaupp, T. Pieber, R. Hovorka, Insulin kinetics in type-1 diabetes: Continuous and bolus delivery of rapid 
acting insulin, IEEE Transactions on Biomedical Engineering 52 (1) (2005) 3-12. doi:10.1109/TBME. 2004.839639.

URL http://ieeexplore.ieee.org/lpdocs/epic03/wrapper.htm? arnumber $=1369583$

[27] J. Wong, J. G. Chase, C. E. Hann, G. M. Shaw, T. F. Lotz, J. Lin, A. J. Le Compte, A subcutaneous insulin pharmacokinetic model for computer simulation in a diabetes decision support role: Model structure and parameter identification, Journal of diabetes science and technology (Online) 2 (4) (2008) 658-671.

URL http://www.ncbi.nlm.nih.gov/pmc/articles/PMC2769764/

[28] M. Vilkhovoy, A control-relevant model of subcutaneous insulin absorption, 2014, pp. 10988-10993. doi:10.3182/20140824-6-ZA-1003.02360 URL http://www.ifac-papersonline.net/Detailed/68759.html

[29] E. W. Kraegen, D. J. Chisholm, Insulin responses to varying profiles of subcutaneous insulin infusion: kinetic modelling studies, Diabetologia 26 (3) (1984) 208-213.

URL http://link. springer . com/article/10.1007/BF00252409

[30] T. Kobayashi, S. Sawano, T. Itoh, K. Kosaka, H. Hirayama, Y. Kasuya, The pharmacokinetics of insulin after continuous subcutaneous infusion or bolus subcutaneous injection in diabetic patients, Diabetes 32 (4) (1983) $331-336$.

URL http://diabetes.diabetesjournals.org/content/32/4/331. short

[31] C. A. Hedman, T. Lindstrom, H. J. Arnqvist, Direct comparison of insulin lispro and aspart shows small differences in plasma insulin profiles after subcutaneous injection in type 1 diabetes, Diabetes Care 24 (6) (2001) 1120-1121. doi:10.2337/diacare.24.6.1120.

URL http://care.diabetesjournals.org/cgi/doi/10.2337/diacare. 24.6 .1120 
[32] J. Plank, A. Wutte, G. Brunner, A. Siebenhofer, B. Semlitsch, R. Sommer, S. Hirschberger, T. R. Pieber, A direct comparison of insulin aspart and insulin lispro in patients with type 1 diabetes, Diabetes care 25 (11) (2002) 2053-2057.

URL http://care.diabetes journals .org/content/25/11/2053.short

[33] H. Akaike, A new look at the statistical model identification, Automatic Control, IEEE Transactions on 19 (6) (1974) 716-723.

URL http://ieeexplore.iee.org/xpls/abs_all.jsp?arnumber= 1100705

[34] K. P. Burnham, Model selection and multimodel inference: a practical information-theoretic approach, 2nd Edition, Springer, New York, 2002.

[35] J. Lin, N. N. Razak, C. G. Pretty, A. Le Compte, P. Docherty, J. D. Parente, G. M. Shaw, C. E. Hann, J. Geoffrey Chase, A physiological intensive control insulin-nutrition-glucose (ICING) model validated in critically ill patients, Computer Methods and Programs in Biomedicine 102 (2) (2011) 192-205. doi:10.1016/j.cmpb.2010.12.008.

URL http://linkinghub.elsevier.com/retrieve/pii/ S0169260710003007

[36] J. G. Chase, G. M. Shaw, J. Lin, C. V. Doran, C. Hann, M. B. Robertson, P. M. Browne, T. Lotz, G. C. Wake, B. Broughton, Adaptive bolus-based targeted glucose regulation of hyperglycaemia in critical care, Medical Engineering \& Physics 27 (1) (2005) 1-11. doi:10.1016/j.medengphy.2004.08.006.

URL http://www.sciencedirect.com/science/article/pii/ S1350453304001420

[37] R. Hovorka, L. J. Chassin, M. Ellmerer, J. Plank, M. E. Wilinska, A simulation model of glucose regulation in the critically ill, Physiological Measurement 29 (8) (2008) 959-978. doi:10.1088/0967-3334/29/8/008 
URL http://stacks.iop.org/0967-3334/29/i=8/a=008?key= crossref.01e3422e8f700f6f33ef42c4d4b946d3

[38] Roche Diagnostics Corporation, Evaluation report: ACCU-CHEK(r) inform II test strips (2012).

[39] A. Wächter, L. T. Biegler, On the implementation of an interior-point filter line-search algorithm for large-scale nonlinear programming, Mathematical Programming 106 (1) (2006) 25-57. doi:10.1007/s10107-004-0559-y. URL http://link. springer.com/10.1007/s10107-004-0559-y

[40] W. E. Hart, J.-P. Watson, D. L. Woodruff, Pyomo: modeling and solving mathematical programs in python, Mathematical Programming Computation 3 (3) (2011) 219-260. doi:10.1007/s12532-011-0026-8. URL http://link. springer.com/10.1007/s12532-011-0026-8

[41] U. M. Ascher, Computer methods for ordinary differential equations and differential-algebraic equations, Society for Industrial and Applied Mathematics, Philadelphia, 1998.

[42] J. C. Boyd, D. E. Bruns, Performance requirements for glucose assays in intensive care units, Clinical Chemistry 60 (12) (2014) 1463-1465. doi: 10.1373/clinchem.2014.231258.

URL http://wWw. clinchem. org/content/60/12/1463 